\title{
Effect of mass distribution on curving performance for a loaded wagon
}

\author{
Duo Zhang $\cdot$ Yinying Tang $\cdot$ Qiyuan Peng $\cdot$ Chunjiao Dong $\cdot$ Yunguang Ye $\mathbb{i}$
}

Received: 24 February 2020/Accepted: 17 March 2021/Published online: 25 March 2021

(C) The Author(s) 2021

\begin{abstract}
The location of wagon gravity center for a loaded wagon is underestimated in a vehicle-track coupled system. The asymmetric wheel load distribution due to loading offset significantly affects the wheel-rail contact state and seriously deteriorates the curving performance in conjunction with the height of gravity center and cant deficiency. Optimizing the location of gravity center and cruising velocity, therefore, is of interest to prevent the derailment and promote the transport capacity of railway wagons. This study aims to reveal the three-dimensional influencing mechanism of mass distribution on vehicle curving performance under different velocities. The wheel unloading ratio is regarded as the evaluation
\end{abstract}

D. Zhang $\cdot$ Y. Tang $\cdot$ Q. Peng

School of Transportation and Logistics, Southwest

Jiaotong University, Chengdu 610031, China

D. Zhang · Y. Tang $\cdot$ Q. Peng

National United Engineering Laboratory of Integrated and Intelligent Transportation, Southwest Jiaotong University, Chengdu 610031, China

C. Dong

Key Laboratory of Transport Industry of Big Data Application Technologies for Comprehensive Transport, Ministry of Transport, Beijing Jiaotong University, Beijing 100044, China

Y. Ye $(\bowtie)$

Institute of Land and Sea Transport Systems, Technical University of Berlin, 10587 Berlin, Germany

e-mail: yunguang.ye@campus.tu-berlin.de index. A simplified quasi-static model is established considering essential assumptions to highlight the influence of lateral and vertical offset on curving performance. For a more accurate description, the MBS models with various locations of wagon gravity center are built and then negotiate curves in different simulation cases. The simulation results reveal that the distribution of wheel unloading ratio determined by loading offset is like contour lines of 'basin'. Based on the conclusions of quasi-static analysis and dynamics simulations, the regression equation is proposed and the fitting parameters are calculated for each simulation case. This paper demonstrates the necessity of optimizing the location of wagon gravity center according to the running condition and offers a novel strategy to load and transport the cargo by railway wagons.

Keywords Railway wagon - Mass distribution Curving performance - Quasi-static analysis . Dynamics simulation $\cdot$ Regression equation

\section{Introduction}

Symmetric distribution is the basic criterion for the loading of cargo on wagons. It has been a consensus that the optimal location of cargo gravity center is at the center of the vehicle laterally and longitudinally. 
Thus, the general wagon-rail models assume that the wagon gravity center (WGC) is the same as its geometry center in the horizontal plane [1-5]. Since the uneven mass distribution could result in an obvious unbalance of wheel load and deteriorate the curving performance seriously, symmetric loading is the basic prerequisite in the studies with respect to guaranteeing wagon running safety [6-11].

However, according to practical experience, skew loading cannot be avoided completely. For the sake of vehicle running safety, loading guidelines of several rail organizations are promulgated to specify the allowed offset values:

(1) International Union of Railways (UIC) [12] The ratio of masses per bogie should be less than $3: 1$ and the ratio of load between the wheels (left/right) of a given axle should be less than 1.25:1. Moreover, the mass per axle should not exceed the maximum axle load.

(2) The Association of American Railroads (AAR) [13]

Longitudinally, the center of load weight should have a certain distance from either truck center, which depends on the ratio between load weight and load limit. Laterally, the load must be located to equalize the weight.

(3) Chinese Railways (CR) [14]

The transversal distance between the cargo's gravity center and the carbody's geometrical center should be within $100 \mathrm{~mm}$. The difference between the masses per bogie should be no more than 10 ton, and the mass of cargo on either bogie should not exceed half of the loading capacity of the wagon.

Depending on these three representative examples, we can recognize that there are no universal requirements on the mass distribution for a loaded wagon. Moreover, the allowed offset values stated in the loading guidelines are sketchy and empirical to some extent. For a further supplement to loading guidelines, the method of dynamics simulation has been used to search for the safe range of wagon's gravity center. Shatunov and Shvets [15] proposed that, as for a kind of 4-axle flat wagon, the maximum lateral offset could reach $150 \mathrm{~mm}$ and longitudinal offset could be expanded too. Bao et al. [16] focused on a common open-top wagon in China and demonstrated that CR criteria were conservative.
The loading guidelines and limited former studies are based on assumptions that the best location of the cargo is at the center of a wagon and the height of WGC should be as low as possible. There are two confusions brought by the aforementioned documents and their prerequisites:

(1) The location of the combined center of gravity is a three-dimensional variable. The decision on skew loading should be made combined with the height of gravity center, which is neglected when defining the allowed offset. Matsumoto et al. [17] and Bekele [18] pointed out that lowering the height of gravity center was an obvious advantage for running safety. Zhang et al. [19] attempted to figure out the threedimensional constraint for the combined center of gravity of a loaded wagon but failed to draw a quantitative conclusion.

(2) The assumption of optimal choice is not convincible. Suda et al. [20,21] proposed that the asymmetric truck may be better for curving performance. Keropyan et al. [22] demonstrated that a longitudinal offset was necessary for the locomotive to promote its traction ability. Since the advantage of asymmetric loading had been proved in other areas, we can infer that symmetric distribution might not be a perfect plan for all the loading cases.

Since loading guidelines and former studies have obvious limitations, this paper analyzes the stereoscopic influence mechanism of mass distribution for a loaded wagon on its curving performance. It has been demonstrated that the safety indices would be severe when the wagon negotiates the transition curve (TC) [19]. Optimizing the location of WGC on TC is necessary to improve the curving performance. However, because of the geometry and changing elevation of TC, it is difficult to reveal the quantitative relationship between the location of WGC and the curving performance on TC. Thus, the process of negotiating the circular curve is the objective of this study.

In Sect. 2, a simplified quasi-static analysis of an uneven loaded wagon is conducted considering profuse assumptions. Section 3 establishes the MBS model and carries out simulations to discuss the qualitative relationship between the location of WGC and the selected safety criterion. Based on the 
conclusions in Sect. 3 and Sect. 4 proposes the regression equation and calculates the fitting parameters for each simulation case.

\section{Analysis of the vehicle curving performance with a simplified quasi-static model}

\subsection{Quasi-static model}

This paper focuses on the two-axle wagon with threepiece bogies. There is no doubt that the suspension system plays a key role in the vehicle running performance by means of weakening the impact between the carbody and wheelset. Nevertheless, as for the unevenly loaded wagon, the suspension device has little effect on inhibiting the unbalanced wheel load which is resulted from the asymmetric mass distribution and closely related to the vehicle running safety. Thus, the quasi-static model is established to highlight the influence of mass distribution on the wheel load. The wagon in uniform circular motion is deemed as suffering equilibrium force including the centrifugal force so that it can be regarded as a whole. In order to simplify the model, essential assumptions are put forward as below:

(1) All the parts of the model are rigid bodies.

(2) The track is smooth and has no rail cant.

(3) The wheelset is symmetric with the center line of the track.

(4) The load of a truck is distributed evenly to each wheelset.

(5) The carbody is at its original position.

The mass distribution is directly related to the vertical contact force. Consequently, the wheel unloading ratio is more sensitive than the derailment coefficient for this issue in terms of definitions. This conjecture was demonstrated by Zhang et al. [19]. Thus, the quasi-static analysis emphatically reveals the vertical contact force for each wheelset and regards the wheel unloading ratio as the evaluation index.

In the $\mathrm{X}-\mathrm{Z}$ plane of the coordinate system of track centerline, the wagon can be regarded as a simply supported beam as is shown in Fig. 1.

where $P_{i}(i=1,2,3,4)$ is the vertical contact force of wheelset; $l$ is half of the length between bogie pivot centers; $G_{z}$ and $L_{z}$ are the vertical component force of wagon's gravity and centrifugal force, respectively;

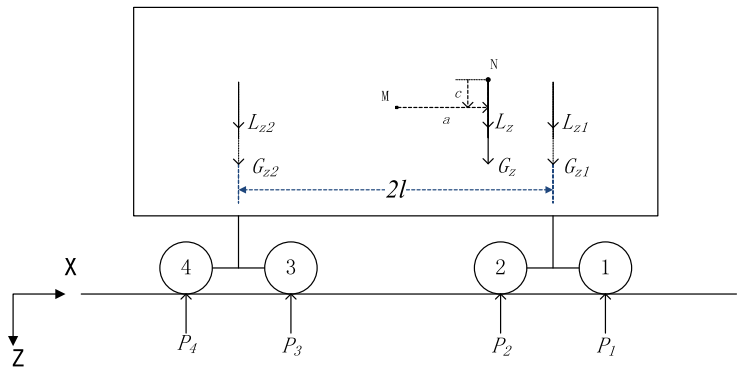

Fig. 1 Longitudinal mass distribution of a loaded wagon

$G_{z q}(q=1,2)$ is distributed $G_{z}$ on each truck; $L_{z q}$ $(q=1,2)$ is distributed $L_{z}$ on each truck; $\mathrm{M}$ and $\mathrm{N}$ are the geometrical center of the carbody and the gravity center of the wagon, respectively; $a$ is the longitudinal offset; $c$ is the vertical offset.

Since the wagon suffers vertical equilibrium force, the sum of vertical contact force can be given by:

$\sum_{i=1}^{4} P_{i}=G_{z}+L_{z}$

Based on the torque balance, the sum of torques around the rear truck center can be presented as follows:

$\left(P_{1}+P_{2}\right) \times 2 l=\left(G_{z}+L_{z}\right) \times(l+a)$.

According to the assumptions, $P_{1}=P_{2}, P_{3}=P_{4}$. Therefore, the vertical contact force can be calculated based on Eqs. (1) and (2):

$P_{1}=P_{2}=\frac{\left(G_{z}+L_{z}\right)(l+a)}{4 l}$

$P_{3}=P_{4}=\frac{\left(G_{z}+L_{z}\right)(l-a)}{4 l}$.

Furthermore, as a simply supported beam, the gravity force and centrifugal force are distributed on the front and rear center plate as below:

$\left\{\begin{array}{l}G_{z 1}=\frac{G_{z}(l+a)}{2 l} \\ G_{z 2}=\frac{G_{z}(l-a)}{2 l} \\ L_{z 1}=\frac{L_{z}(l+a)}{2 l} \\ L_{z 2}=\frac{L_{z}(l-a)}{2 l}\end{array}\right.$

The mass of wagon is distributed to each truck longitudinally as already stated. Then, lateral offset 
assigns the load of each wheelset to the wheels as Fig. 2 illustrates.

where $G_{q}$ and $L_{q}$ are the gravity and centrifugal force of the mass loaded on the truck whose order is $q$, respectively; subscript $q$ denotes the front truck when its value is 1 and the rear truck when its value is $2 ; P$ is the vertical contact force; $Q$ is the lateral contact force; subscript $i$ denotes the order of wheelset as is shown in Fig. 1; subscript $l$ and $r$ denote the left and right wheel of the wheelset, respectively; $\alpha$ represents the angle resulted from superelevation; $b$ is the lateral offset; $c$ is the vertical offset from $\mathrm{N}$ to $\mathrm{M} ; h$ is the vertical distance from $\mathrm{M}$ to the top of rail; $d$ is the half of tape circle distance. In this paper, we take the right-hand curve as an example. It is easy to point out that:

$\left\{\begin{array}{l}i=1,2 q=1 \\ i=3,4 \quad q=2\end{array}\right.$.

As for the truck whose order is $q(q=1,2)$, its distributed vertical load is borne by the corresponding wheelsets:

$\sum P_{i l}+\sum P_{i r}=G_{z q}+L_{z q}$.

Moreover, the resultant moment of the truck can be calculated as:

$\sum P_{i r} \times 2 d+\left(L_{y q}-G_{y q}\right) \times(h+c)$
$=\left(L_{z q}+G_{z q}\right) \times(d+b)$.

Based on Eqs. (6) and (7), the vertical contact force can be presented as:

$P_{i l}=\frac{\left(L_{z q}+G_{z q}\right)(d-b)+\left(L_{y q}-G_{y q}\right)(h+c)}{4 d}$

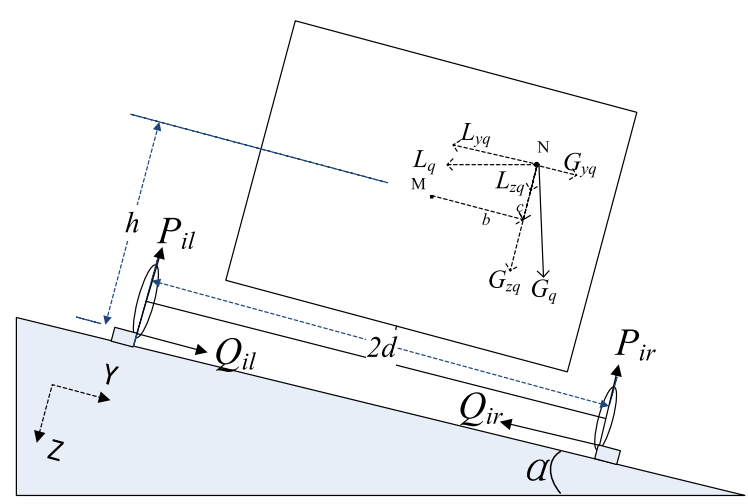

Fig. 2 Lateral mass distribution of loaded wagon
$P_{i r}=\frac{\left(L_{z q}+G_{z q}\right)(d+b)-\left(L_{y q}-G_{y q}\right)(h+c)}{4 d}$.

For any wheelset, the wheel unloading ratio (UN) can be derived as:

$\frac{P_{i l}-P_{i r}}{P_{i l}+P_{i r}}=\frac{L_{y q}-G_{y q}}{L_{z q}+G_{z q}} \times \frac{h+c}{d}-\frac{b}{d}$.

Combined with the geometric relationship, Eq. (11) can be derived as:

$\frac{P_{i l}-P_{i r}}{P_{i l}+P_{i r}}=\frac{L_{z q}-G_{z q} \tan ^{2} \alpha}{\tan \alpha\left(L_{z q}+G_{z q}\right)} \times \frac{h+c}{d}-\frac{b}{d}$.

Since we have assumed that the truck would allocate its load to inclusive wheelsets evenly, wheelsets of the same truck have identical UN. According to Eq. (10), it seems that the mass loaded on truck has effect on the UN. For revealing the effect of the mass distribution significantly, Eq. (5) is substituted to Eq. (12) to obtain:

$$
\begin{aligned}
\frac{P_{i l}-P_{i r}}{P_{i l}+P_{i r}} & =\frac{L_{z}-G_{z} \tan ^{2} \alpha}{\tan \alpha\left(L_{z}+G_{z}\right)} \times \frac{h+c}{d}-\frac{b}{d} \\
& =\frac{v^{2}-g R \tan \alpha}{v^{2} \tan \alpha+g R} \times \frac{h+c}{d}-\frac{b}{d}
\end{aligned}
$$

where $v$ denotes the vehicle running velocity; $R$ denotes the radii of curve; $g$ denotes the acceleration of gravity.

\subsection{Analysis of derivative results}

Equation (11) illustrates that apart from the lateral and vertical offset, cant deficiency affects the value of UN significantly. The values of $(h+c), d$ and $\left(L_{z q}+G_{z q}\right)$ are definitely positive. Thus, the positive/negative signs of $\left(L_{y q}-G_{y q}\right)$ and $b$ determine the trend of change in the absolute value of UN as below:

(1) $L_{y q}<G_{y q}, b>0$ or $L_{y q}>G_{y q}, b<0$

The absolute value of UN is positively correlated with $|b|$ and $c$.

(2) $L_{y q}<G_{y q}, b<0$ or $L_{y q}>G_{y q}, b>0$

It is complicated to describe the trend of the absolute value of $\mathrm{UN}$ since it is denoted as the sum of a positive expression and a negative expression containing variables. Numerical computation is needed to reveal the distribution rules of UN. 
The difference between the lateral component forces of centrifugal force and gravity of distributed mass reflects cant deficiency, which is derived to obtain Eq. (13). Based on Eq. (13), we can demonstrate the relationship among the loading offset, velocity and UN clearly. As an example, we assume that $\tan \alpha$ equals 0.1045 , the gauge equals $1435 \mathrm{~mm}$, $d$ equals $0.75 \mathrm{~m}$. For a better description, we use the variable of $z$ to denote the sum of $h$ and $c$ and set the scopes of $z$ and $b$. When the loaded wagon negotiates curves whose radii are $350 \mathrm{~m}$ and $600 \mathrm{~m}$, respectively, with velocities from $10 \mathrm{~m} / \mathrm{s}$ to $25 \mathrm{~m} / \mathrm{s}$, the absolute value of UN can be calculated as Fig. 3 illustrates.

Figure 3 reveals the influence of loading offset and velocity on UN that can be concluded as below:

(1) For the selected velocity and curve radius, the distribution of UN is similar as the contour lines

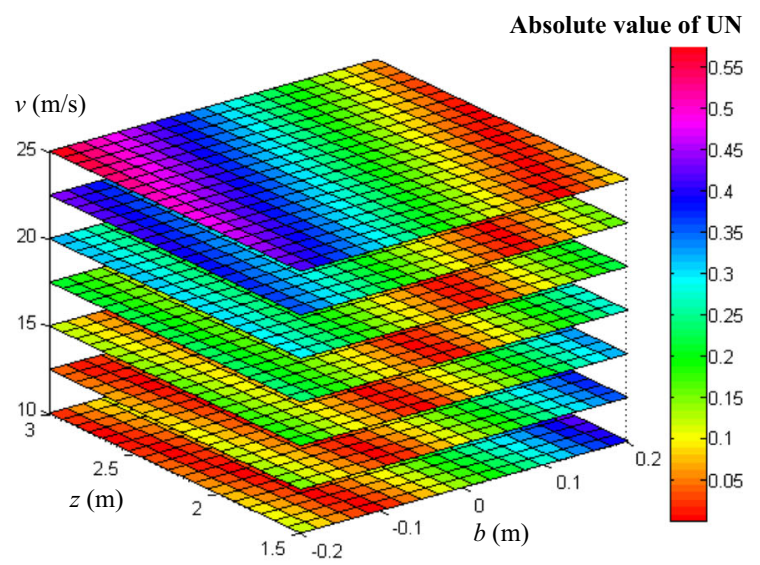

(a) $R=350 \mathrm{~m}$

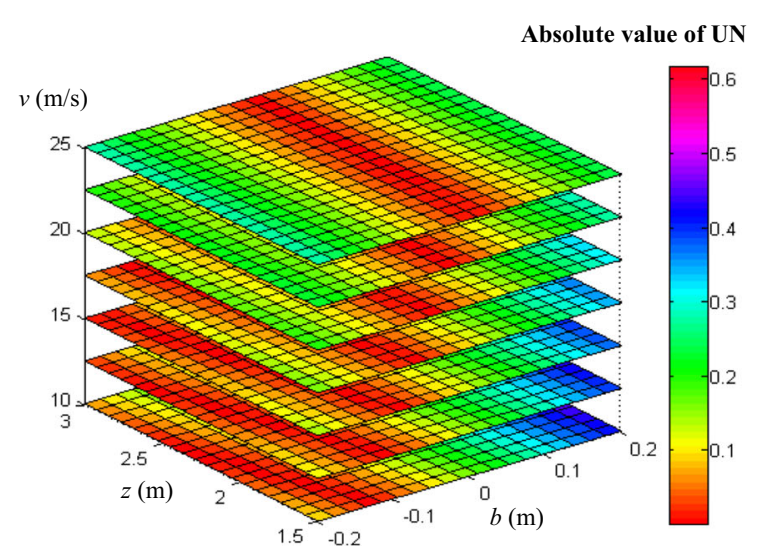

(b) $R=600 \mathrm{~m}$

Fig. 3 Distribution of the absolute value of UN of 'basin.' With the increase in velocity, the location of the basin moves to the right of the wagon.

(2) For most locations, the absolute value of UN increases with the increase in vertical offset. But if there is a large negative offset laterally, the absolute value of UN will decrease as the vertical offset increases when the velocity is low.

(3) The balancing velocity $(\mathrm{m} / \mathrm{s})$ can be calculated as Eq. (14):

$V_{0}=\sqrt{g R \tan \alpha}$.

For curves whose radii are $350 \mathrm{~m}$ and $600 \mathrm{~m}$, their balancing velocities are $19 \mathrm{~m} / \mathrm{s}$ and $25 \mathrm{~m} /$ $\mathrm{s}$, respectively, based on Eq. (14). Figure 3 demonstrates that when the wagon negotiates the curve at the balancing velocity, UN can be constrained efficiently with the fluctuations of lateral and vertical offset.

By means of quasi-static analysis, the roles of lateral and vertical offset in UN can be revealed. In order to demonstrate the conclusions of quasi-static analysis and study the effect of longitudinal offset on UN, dynamics simulation should be implemented.

\section{Dynamics simulation}

\subsection{Dynamic equations of carbody}

In this paper, we adopt $\mathrm{C}_{70 \mathrm{H}}$ as the analysis object, which is one of the commonly used open-top cars in China. The MBS model of $\mathrm{C}_{70 \mathrm{H}}$ is made up of cargo, carbody and two three-piece bogies. The schematic diagram is shown in Fig. 4.

where $H_{j}(j=1,2,3)$ denotes the distance between the gravity centers of different components; $r$ denotes the rolling radius of the wheel; $i$ denotes the angle resulted from the superelevation; $\alpha$ denotes the tilt angle of carbody; $s$ denotes half of the tape circle distance.

Due to the loading offset, the dynamic equations of carbody play a primary role in building the MBS model of $\mathrm{C}_{70 \mathrm{H}}$. Because it is deemed that the bolster is fixed with carbody in each degree of freedom except roll, the carbody can be regarded as being exerted by the lateral and vertical forces of the secondary 


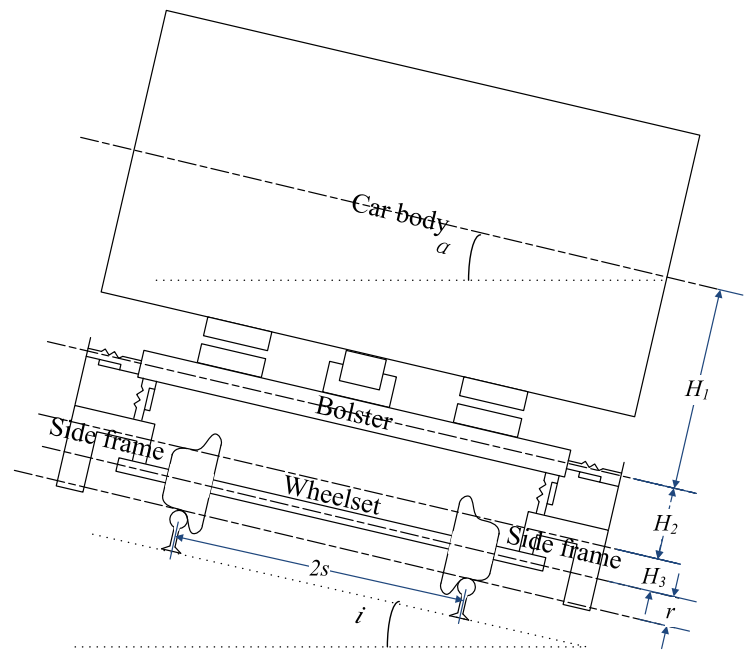

Fig. 4 Side view of $\mathrm{C}_{70 \mathrm{H}}$

suspensions, as well as the resistance moments of the bolster around Y-axis. The force condition of the carbody is illustrated in Fig. 5.

where $O$ denotes the geometrical center of carbody; $C$ denotes the gravity center of carbody; $m_{C}$ denotes the mass of carbody; $m_{B}$ denotes the mass of bolster; $P_{i}(i=1,2,3,4)$ denote the equivalent operating points of the secondary suspensions; $F_{y i}(i=1,2,3,4)$ and $F_{z i}(i=1,2,3,4)$ denote the lateral and vertical forces of secondary suspensions, respectively; $M_{B i}(i=1,2)$ denote the moments around the Z-axis of center plates; $M_{z i}(i=1,2,3,4)$ denote the moments around the Z-axis of side bearers; $M_{y i}(i=1,2,3,4)$ denote the moments around the Y-axis of bolsters; $F_{L C}$ denotes the inertial force on carbody; $F_{L B i}(i=1,2)$ denote the inertial forces on bolsters; $Y_{C}$ and $Z_{C}$ denote the lateral and vertical distances of carbody,

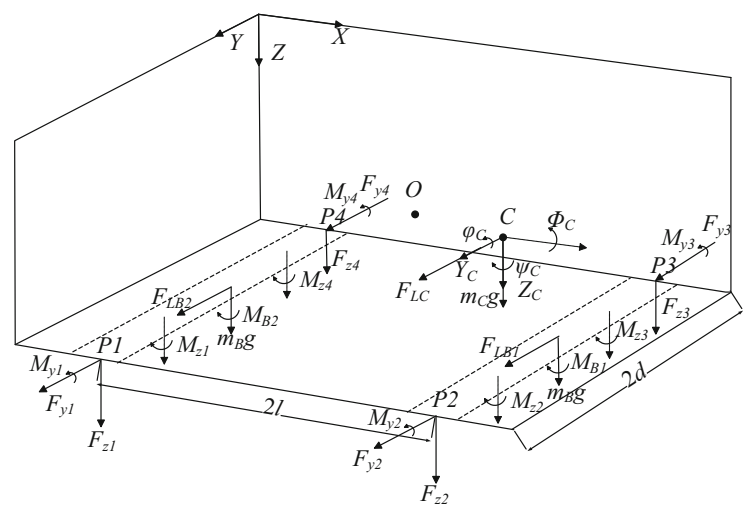

Fig. 5 Forces and moments on the carbody respectively; $\phi_{C}, \varphi_{C}$ and $\psi_{C}$ denote the roll angle, pitch angle and yaw angle, respectively.

In order to simplify the issue, we define that $\overrightarrow{O C}=(x, y,-z)$. We assume that the curve radius is $R$ and the speed of wagon is $v$. Then the curve radius corresponding to $C$ is $(R-y)$. The rotational inertia of carbody can be represented by $I_{C x}, I_{C y}$ and $I_{C z}$. The rotational inertia of bolster can be represented by $I_{B x}$, $I_{B y}$ and $I_{B z}$. Using the symbols shown in Figs. 4 and 5, the dynamic equations of the carbody in the coordinate system of track can be expressed as follows:

$$
\left\{\begin{array}{l}
\sum_{i=1}^{4} F_{y i}+\left(m_{C}+2 m_{B}\right) g \alpha=m_{C}\left[\ddot{Y}_{C}+\frac{v^{2}}{R-y}+\ddot{\alpha}\left(z+H_{1}+H_{2}+H_{3}+r\right)\right] \\
\quad+2 m_{B}\left[\ddot{Y}_{C}-H_{1} \ddot{\phi}_{C}+\frac{v^{2}}{R}+\ddot{\alpha}\left(H_{2}+H_{3}+r\right)\right] \\
\sum_{i=1}^{4} F_{z i}+\left(m_{C}+2 m_{B}\right) g=m_{C}\left[\ddot{Z}_{C}+\frac{v^{2}}{R-y} \alpha-\ddot{\alpha}(s-y)\right]+2 m_{B}\left(\ddot{Z}_{C}+\frac{v^{2}}{R} \alpha-\ddot{\alpha} s\right) \\
\quad-\sum_{i=1}^{4} F_{y i}\left(H_{1}+z\right)+\left(F_{z 1}+F_{z 2}\right)(d-y)-\left(F_{z 3}+F_{z 4}\right)(d+y) \\
=\left[I_{C x}+2 I_{B x}+2 m_{B}\left(H_{1}+z\right)^{2}\right]\left(\ddot{\phi}_{C}+\ddot{\alpha}\right) \\
\sum_{i=1}^{4} M_{y i}+\left(F_{z 1}+F_{z 4}\right)(l+x)-\left(F_{z 2}+F_{z 3}\right)(l-x) \\
=\left[I_{C y}+2 I_{B y}+m_{B}(l+x)^{2}+m_{B}(l-x)^{2}\right] \ddot{\varphi}_{C} \\
\sum_{i=1}^{4} M_{z i}+\sum_{i=1}^{2} M_{B i}+\left(F_{y 2}+F_{y 3}\right)(l-x)-\left(F_{y 1}+F_{y 4}\right)(l+x) \\
=I_{C z}\left[\ddot{\psi}_{C}+v \frac{\mathrm{d}}{\mathrm{d} t}\left(\frac{1}{R-y}\right)\right] .
\end{array}\right.
$$

Based on Eq. (15) and the former studies about the dynamic equations of freight bogies [23, 24], the MBS model of $\mathrm{C}_{70 \mathrm{H}}$ can be established.

\subsection{Description of MBS model}

The cargo and carbody are both modeled as rigid bodies and connected by a fixed joint so as to be regarded as a whole. The wagon has two bogies, and each bogie is made up of one bolster, one center plate, two wheelsets, two side bearers, two side frames, and four axleboxes. These bodies are connected by forces, joints, and constraints, including primary suspension forces, secondary suspension forces, etc. The wheelset adopts LM tread profile that matches the rail profile of UIC60 as Fig. 6 shows.

The axlebox is connected with the wheelset by a revolute joint. The primary suspension, denoted by bistops which can represent the contact force between the adapter and guiding frame, links the axlebox to the side frame that is connected with the bolster by the secondary suspension. For $\mathrm{C}_{70 \mathrm{H}}$, its secondary suspension is also linked to two wedges which are 


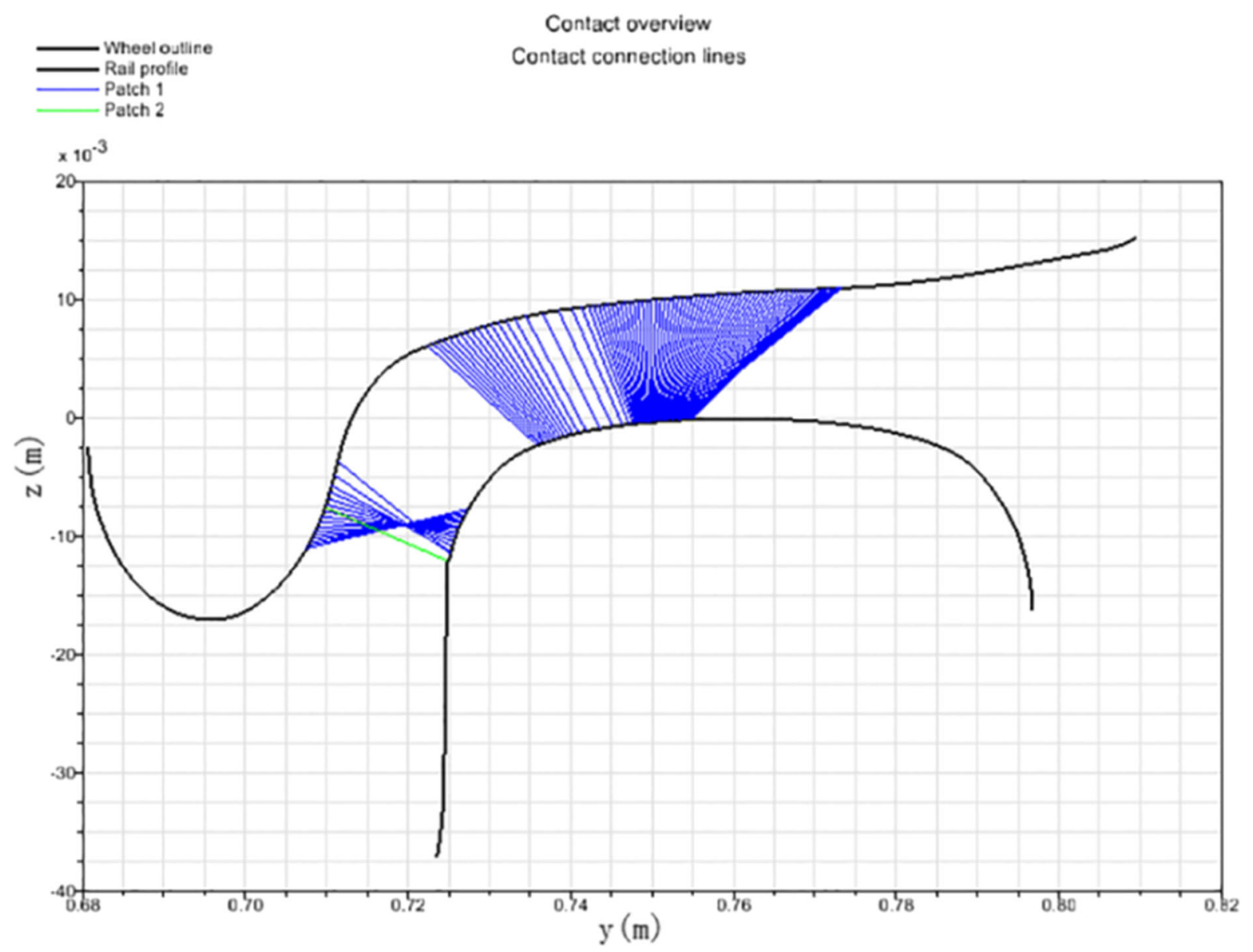

Fig. 6 Profile of LM tread (Flange angle is $70^{\circ}$ ) and UIC60 (rail cant is $1 / 40$ )

between the bolster and side frame to offer normal force and planar friction on the inclined plane and vertical plane of each wedge in order to decrease the vibration [7]. Between the carbody and the bolster, there is the side bearer which is represented by a spring with gap and the center plate which offers contact force, friction force, and torque component around the normal. The key parameters of the MBS model are listed in Table 1.

\subsection{Loading cases and simulation cases}

In this paper, the cargo and carbody are connected with the fixed joint so as to be regarded as a whole. The procedure of dynamics simulation is to update the model by varying the location of the cargo and run each model through the tracks of different radii with various velocities.

\subsubsection{Loading cases}

The location of WGC contains longitudinal offset $(x)$, lateral offset $(y)$ and the vertical distance from WGC to the top of rail $(z)$. According to the loading guidelines enumerated in Sect. 1, there is no uniform requirement to define the maximum value of $x$. We think the most basic requirement is that the mass of cargo on either bogie should not exceed half of the load limit $[13,14]$. We use $M_{\text {limit }}$ to indicate the load limit and use $M_{\text {empty }}$ to indicate the mass of empty wagon. As is shown in Fig. 1 where the value of $x$ is represented as $a$, the load distributed on each bogie should be no more than $\left(M_{\text {limit }}+M_{\text {empty }}\right) / 2$. Considering the parameter values shown in Table 1 , the maximum value of $x$ can be calculated as $1.2 \mathrm{~m}$. Then, the values of $x$ and the corresponding locations of the 
Table 1 Primary characteristics of the MBS model

\begin{tabular}{|c|c|}
\hline Parameters & Value \\
\hline Empty wagon mass (kg) & $2.38 \mathrm{E}+04$ \\
\hline Carbody rolling moment of inertia $\left(\mathrm{kg} \mathrm{m}^{2}\right)$ & $1.50 \mathrm{E}+04$ \\
\hline Carbody pitching moment of inertia $\left(\mathrm{kg} \mathrm{m}^{2}\right)$ & $2.14 \mathrm{E}+05$ \\
\hline Carbody yawing moment of inertia $\left(\mathrm{kg} \mathrm{m}^{2}\right)$ & $2.24 \mathrm{E}+05$ \\
\hline Load limit stenciled on the car $(\mathrm{kg})$ & $7 \mathrm{E}+4$ \\
\hline Cargo mass $(\mathrm{kg})$ & $5.00 \mathrm{E}+04$ \\
\hline Cargo rolling moment of inertia $\left(\mathrm{kg} \mathrm{m}^{2}\right)$ & $1.36 \mathrm{E}+04$ \\
\hline Cargo pitching moment of inertia $\left(\mathrm{kg} \mathrm{m}^{2}\right)$ & $8.17 \mathrm{E}+04$ \\
\hline Cargo yawing moment of inertia $\left(\mathrm{kg} \mathrm{m}^{2}\right)$ & $8.70 \mathrm{E}+04$ \\
\hline Vertical distance from empty wagon gravity center to top of rail (m) & 1.085 \\
\hline Length of carbody (m) & 13 \\
\hline Width of carbody (m) & 2.892 \\
\hline Height of carbody (m) & 2.05 \\
\hline Bogie frame mass $(\mathrm{kg})$ & 355 \\
\hline Bogie frame rolling moment of inertia $\left(\mathrm{kg} \mathrm{m}^{2}\right)$ & 15 \\
\hline Bogie frame pitching moment of inertia $\left(\mathrm{kg} \mathrm{m}^{2}\right)$ & 146 \\
\hline Bogie frame yawing moment of inertia $\left(\mathrm{kg} \mathrm{m}^{2}\right)$ & 134 \\
\hline Wheelset rolling radius (m) & 0.42 \\
\hline Wheel base (m) & 1.8 \\
\hline Tape circle distance $(\mathrm{m})$ & 1.493 \\
\hline Axle length $(\mathrm{m})$ & 1.981 \\
\hline Distance between center pivots $(\mathrm{m})$ & 9.21 \\
\hline Wheelset mass $(\mathrm{kg})$ & 1290 \\
\hline Wheelset rolling moment of inertia $\left(\mathrm{kg} \mathrm{m}^{2}\right)$ & 760 \\
\hline Wheelset pitching moment of inertia $\left(\mathrm{kg} \mathrm{m}^{2}\right)$ & 77 \\
\hline Wheelset yawing moment of inertia $\left(\mathrm{kg} \mathrm{m}^{2}\right)$ & 760 \\
\hline Bolster mass $(\mathrm{kg})$ & 652 \\
\hline Bolster rolling moment of inertia $\left(\mathrm{kg} \mathrm{m}^{2}\right)$ & 254.4 \\
\hline Bolster pitching moment of inertia $\left(\mathrm{kg} \mathrm{m}^{2}\right)$ & 19.73 \\
\hline Bolster yawing moment of inertia $\left(\mathrm{kg} \mathrm{m}^{2}\right)$ & 254.4 \\
\hline Primary spring longitudinal stiffness (MN/m) & 80 \\
\hline Primary spring lateral stiffness (MN/m) & 125 \\
\hline Secondary spring longitudinal stiffness (MN/m) & 3.867 \\
\hline Secondary spring lateral stiffness $(\mathrm{MN} / \mathrm{m})$ & 3.867 \\
\hline Secondary spring vertical stiffness (MN/m) & 5.473 \\
\hline
\end{tabular}

cargo's gravity center can be designed as Table 2 illustrates.

Comparatively speaking, the determination for the values of $y$ and $z$ is empirical and rough. They are presented in Tables 3 and 4 . The definitions of $y_{c}$ and $z_{c}$ are similar as $x_{c}$, describing the distance between the reference position and the cargo's center of gravity.
Table 2 Values of $x$ and corresponding values of $x_{c}$

\begin{tabular}{llllllll}
\hline$x(\mathrm{~m})$ & -1.2 & -0.8 & -0.4 & 0 & 0.4 & 0.8 & 1.2 \\
$x_{c}(\mathrm{~m})$ & -1.77 & -1.18 & -0.59 & 0 & 0.59 & 1.18 & 1.77
\end{tabular}

$x_{c}$ denotes the longitudinal distance from the geometrical center of carbody to the cargo's gravity center 
Table 3 Values of $y$ and corresponding values of $y_{c}$

\begin{tabular}{llllllll}
\hline$Y(\mathrm{~m})$ & -0.135 & -0.09 & -0.045 & 0 & 0.045 & 0.09 & 0.135 \\
$y_{c}(\mathrm{~m})$ & -0.2 & -0.13 & -0.07 & 0 & 0.07 & 0.13 & 0.2 \\
\hline
\end{tabular}

Table 4 Values of $z$ and corresponding values of $z_{c}$

\begin{tabular}{|c|c|c|c|c|c|c|c|c|c|c|}
\hline$Z(\mathrm{~m})$ & 1.5 & 1.65 & 1.8 & 1.95 & 2.1 & 2.25 & 2.4 & 2.55 & 2.7 & 2.85 \\
\hline$z_{c}(\mathrm{~m})$ & 1.70 & 1.92 & 2.14 & 2.36 & 2.58 & 2.81 & 3.03 & 3.25 & 3.47 & 3.69 \\
\hline
\end{tabular}

Obviously, there are 490 loading cases based on the values of $x_{c}, y_{c}$ and $z_{c}$. For each loading case, the MBS model would be implemented in each simulation case.

\subsubsection{Simulation cases}

In this paper, the MBS model runs through the righthand curves with different curve radii. Generally speaking, the curve with a small radius is an unfavorable factor to affect vehicle curving performance. We assume that the radii of curves are $350 \mathrm{~m}$ and $600 \mathrm{~m}$, respectively [25].

According to EN14363 and EN 13,803, we set the superelevation, maximum cant deficiency and maximum cant excess as relatively high values, which are $150 \mathrm{~mm}, 130 \mathrm{~mm}$ and $130 \mathrm{~mm}$, respectively [25, 26]. The gauge is designed as $1435 \mathrm{~mm}$. Then the maximum running velocity $\left(V_{\max }\right)$, minimum running velocity $\left(V_{\min }\right)$ and balancing velocity $\left(V_{0}\right)$ can be calculated. The simulation cases consisting of different curve radii and cruising velocities are illustrated in Table 5.

In this section, we adopt the standard track irregularity spectrum of FRA 5 (the 5th class track defined by Federal Railroad Administration of US) as the excitation [27-29].

\subsection{Simulation result and analysis}

In this paper, the wheel unloading radio (UN) is the criterion to evaluate the vehicle curving performance. When the updated MBS model endowed with its loading case negotiates the $120 \mathrm{~m}$ length curve in a simulation case, the maximum absolute value of UN $\left(\mathrm{UN}_{\max }\right)$ for all the wheelsets can be monitored. In this paper, we regard 0.9 as the limit value of UN [24]. For
Table 5 Detail of simulation cases

\begin{tabular}{lll}
\hline Curve radius $(\mathrm{m})$ & Velocity $(\mathrm{m} / \mathrm{s})$ & Case No \\
\hline 350 & $7\left(V_{\min }\right)$ & 1 \\
350 & 13 & 2 \\
350 & $19\left(V_{0}\right)$ & 3 \\
350 & 22 & 4 \\
350 & $26\left(V_{\max }\right)$ & 5 \\
600 & $9\left(V_{\min }\right)$ & 6 \\
600 & 17 & 7 \\
600 & $25\left(V_{0}\right)$ & 8 \\
600 & 30 & 9 \\
600 & $34\left(V_{\max }\right)$ & 10 \\
\hline
\end{tabular}

a certain simulation case, if we delete all the loading cases which would result in the $\mathrm{UN}_{\text {max }}$ larger than 0.9, then the safety range of WGC can be obtained. The distributions of $\mathrm{UN}_{\max }$ for the safety loading cases in each simulation case are illustrated in Fig. 7.

where $R$ denotes the curve radius and $v$ denotes the cruising velocity.

Figure 7 gives us full evidence to draw the conclusions as below:

(1) Under the premise of a constant $z$, the distribution of $\mathrm{UN}_{\max }$ is similar to the contour lines of 'basin.' In the lateral direction, the location of the 'basin' moves to the right side of the wagon when it runs on the same curve track with a higher velocity. In longitudinal direction, there is no obvious law about the location of the 'basin.' Generally, the 'basin' is around the lateral center line or in the front of the wagon.

(2) For the majority of simulation cases, their distributions of $\mathrm{UN}_{\max }$ revealed in Fig. 7 
Fig. 7 Distributions of $\mathrm{UN}_{\max }$ for the safety loading cases

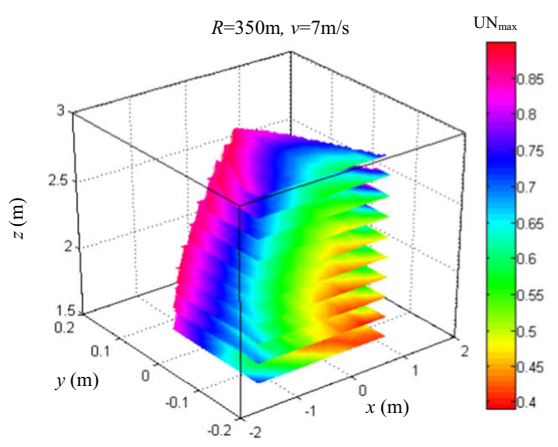

(a) NO.1 simulation case

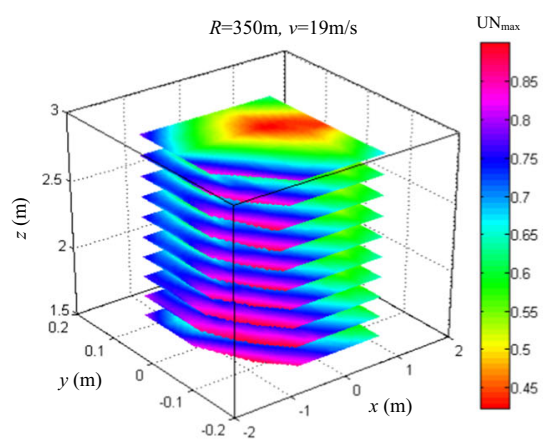

(c) NO.3 simulation case

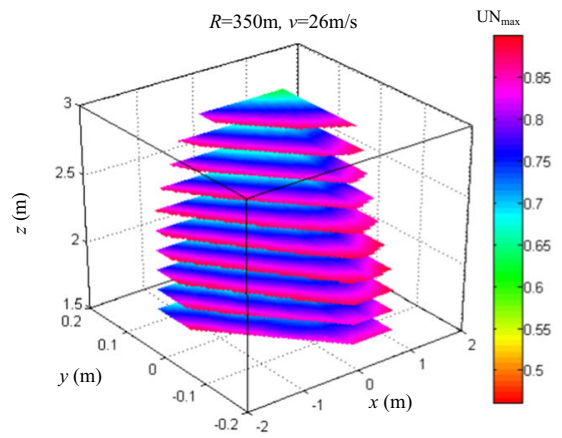

(e) NO.5 simulation case

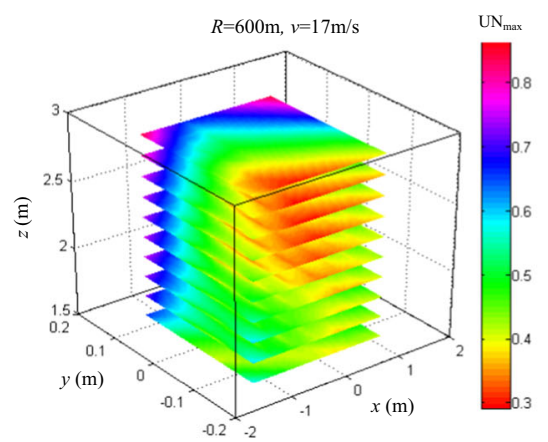

(g) NO.7 simulation case

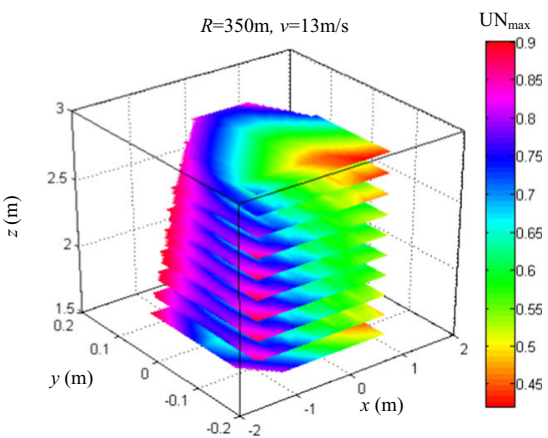

(b) NO.2 simulation case

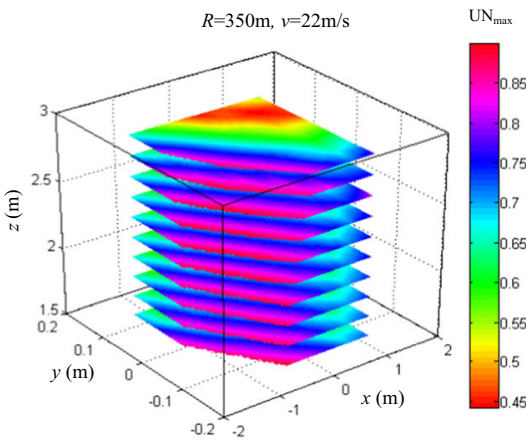

(d) NO.4 simulation case

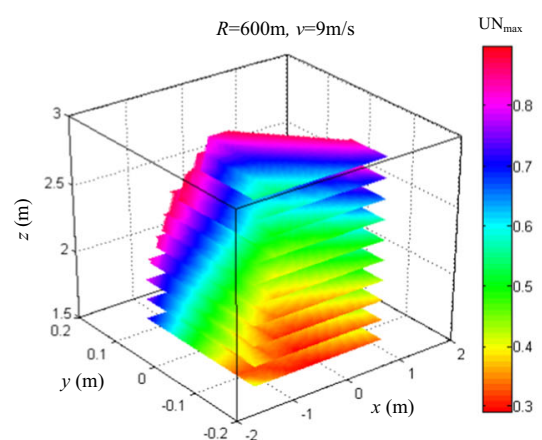

(f) NO.6 simulation case

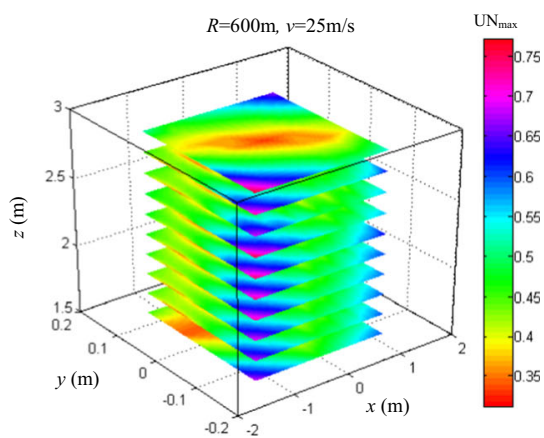

(h) NO.8 simulation case 
Fig. 7 continued

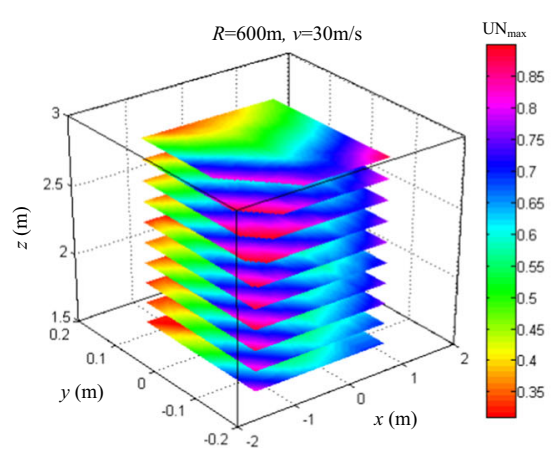

(i) NO.9 simulation case

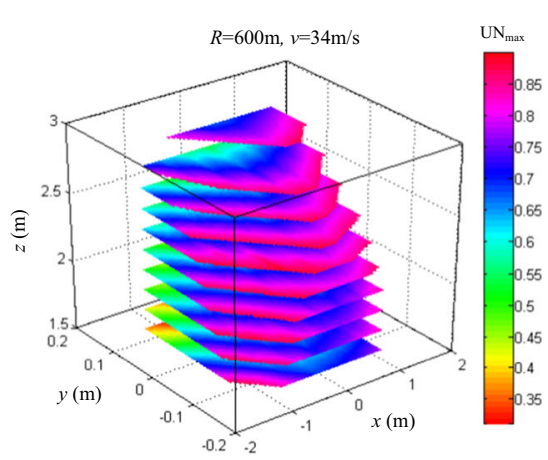

(j) NO.10 simulation case support the consensus that higher WGC will lead to worse curving performance. However, as is illustrated in Fig. 7b, g, $\mathrm{UN}_{\max }$ will decrease with the increase in $z$ when there is a large lateral offset to the left of the wagon. Such an unexpected trend happens when there is a small cant excess. The mechanism of this novel phenomenon can be demonstrated based on Eq. (11) and is revealed in Fig. 3 in Sect. 2.

\section{Regression analysis of simulation data}

The dynamics simulation results revealed in Fig. 7 demonstrate the effect of mass distribution on curving performance in each simulation case. Because every subgraph in Fig. 7 shows a highly unified law, we believe that there could be a common fitting equation between the location of the WGC and $\mathrm{UN}_{\max }$. Based on the characteristics of horizontal distribution illustrated in Fig. 7, we tried using the oblique ellipse equation to denote the role of loading offset in $\mathrm{UN}_{\text {max }}$. Besides, we attempt to draw on Eq. (13) in Sect. 2 to denote the role of vertical distance in $\mathrm{UN}_{\max }$ because the conclusions of the quasi-static analysis are consistent with the vertical distribution characteristic of $\mathrm{UN}_{\max }$ as is illustrated in Fig. 7. For better expression of the equation, we use $p$ to denote $\mathrm{UN}_{\text {max }}$. The regression equation can be proposed as:

$$
\begin{aligned}
p= & \alpha x^{2}+\beta x+\gamma y^{2}+\mu y+\theta x y+\frac{v^{2}-g R \tan \alpha}{v^{2} \tan \alpha+g R} \times z \\
& \times \xi+\eta
\end{aligned}
$$

Table 6 Expressions of RMSE and $R^{2}$

\begin{tabular}{lc}
\hline Indicators & Expression \\
\hline RMSE & $\sqrt{\frac{1}{n} \sum_{i=1}^{n}\left(y_{i}^{*}-y_{i}\right)^{2}}$ \\
$R^{2}$ & $1-\frac{\sum_{i=1}^{n}\left(y_{i}-y_{i}^{*}\right)^{2}}{\sum_{i=1}^{n}\left(y_{i}-y_{i}\right)^{2}}$ \\
\hline
\end{tabular}

$y_{i}$ denotes the original data, $y_{i}^{*}$ denotes the fitting data

where the value of $\tan \alpha$ is 0.1045 in this paper, $g$ is the gravity acceleration, $v$ and $R$ are the constants depending on simulation cases.

Based on the data in each simulation case, the values of parameters can be calculated. For reflecting the fitting effect of Eq. (16), two evaluation indicators are adopted: the root mean squared error (RMSE) and the coefficient of determination $\left(R^{2}\right)$. The definitions of RMSE and $R^{2}$ are shown in Table 6.

The values of parameters and the corresponding indicators for each simulation case are illustrated in Table 7.

In order to be compared with the simulation data intuitively, the fitting data are revealed in Fig. 8.

According to the evaluation indicators shown in Table 7 and the contrast between Figs. 7 and 8, Eq. (16) can basically be considered as the fitting equation and the values of fitting parameters are illustrated in Table 7. Based on the regression results, we can directly assess vehicle curving performance with the consideration of mass distribution or adjust the cruising velocity in order to improve vehicle curving performance. 
Table 7 Regression results

\begin{tabular}{|c|c|c|c|c|c|c|c|c|c|}
\hline Case No & $\alpha$ & $\beta$ & $\gamma$ & $\mu$ & $\theta$ & $\xi$ & $\eta$ & RMSE & $R^{2}$ \\
\hline 1 & 0.117 & -0.019 & 0.254 & 1.395 & 0.329 & -1.673 & 0.324 & 0.045 & 0.880 \\
\hline 2 & 0.083 & -0.046 & 4.915 & 0.860 & 0.590 & -0.041 & 0.599 & 0.052 & 0.780 \\
\hline 3 & 0.081 & -0.122 & 8.089 & -0.691 & 0.677 & -77.177 & 0.655 & 0.032 & 0.915 \\
\hline 4 & 0.075 & -0.144 & 2.750 & -1.389 & 0.502 & 0.816 & 0.560 & 0.032 & 0.937 \\
\hline 5 & 0.056 & -0.137 & -0.344 & -1.386 & -0.052 & 1.570 & 0.498 & 0.023 & 0.960 \\
\hline 6 & 0.059 & 0.041 & 0.349 & 1.459 & -0.027 & -2.947 & 0.021 & 0.033 & 0.956 \\
\hline 7 & 0.050 & 0.033 & 2.767 & 1.268 & 0.229 & 0.659 & 0.553 & 0.042 & 0.895 \\
\hline 8 & 0.059 & 0.005 & 8.961 & -0.302 & 0.571 & 7.265 & 0.326 & 0.033 & 0.874 \\
\hline 9 & 0.074 & -0.025 & 2.864 & -1.456 & 0.402 & 1.751 & 0.296 & 0.041 & 0.919 \\
\hline 10 & 0.083 & -0.056 & -0.135 & -1.626 & 0.239 & 2.402 & 0.186 & 0.040 & 0.926 \\
\hline
\end{tabular}

\section{Conclusion}

Loading offset significantly threatens the running safety of railway wagons. Several major railway organizations have formulated loading guidelines to limit the mass distribution for a loaded wagon, and a few relevant studies were conducted to verify the accuracy of rules. Both the existing regulations and studies focused on the limit value of loading offset without demonstrating the influence mechanism of mass distribution on curving performance threedimensionally. The rigid regulations can meet the basic requirements for running safety, but it has great limitations. On the one hand, vehicle curving performance cannot be promoted by optimizing the location of WGC or running velocity. On the other hand, the limit value of the loading offset is in accordance with the worst running condition. Thus, the transportation capacity of the railway wagon is decreased in normal circumstances.

Based on the above considerations, this paper explores the relationship between the location of WGC and the safety criterion considering different cruising velocities. A simplified quasi-static model is established on the premise of several assumptions to reveal the roles of lateral offset, vertical offset and velocity in the maximum absolute value of the wheel unloading ratio $\left(\mathrm{UN}_{\max }\right)$. MBS models with different loading offsets negotiate curves in various simulation cases. By means of dynamics simulations, the results of the quasi-static analysis are validated and the distribution of $\mathrm{UN}_{\max }$ for each simulation case is presented as the contour lines of 'basin.' According to the conclusions drawn from quasi-static analysis and dynamics simulation, the fitting equation is established. Based on the dynamics simulation data, the fitting parameters are calculated for each simulation case.

This paper proposes a novel strategy to load the cargo and optimize the cruising velocity. Unfortunately, up to now, we haven't been able to build the connection between the value of fitting parameter and curve radius or velocity. Further dynamics simulations may be needed to help us establish a fitting equation applicable for various simulation cases.

Moreover, the track irregularity is taken as the only oscillation source(OS) in this paper. More OS should be considered to make the conclusions more practical. As far as we are concerned, the changing elevation of transition curve, the action of switch and the wind can be modeled as the supplementary OS in the future study. 


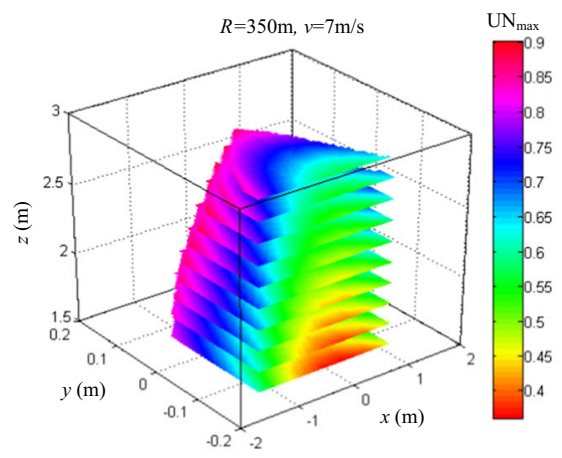

(a) NO.1 simulation case

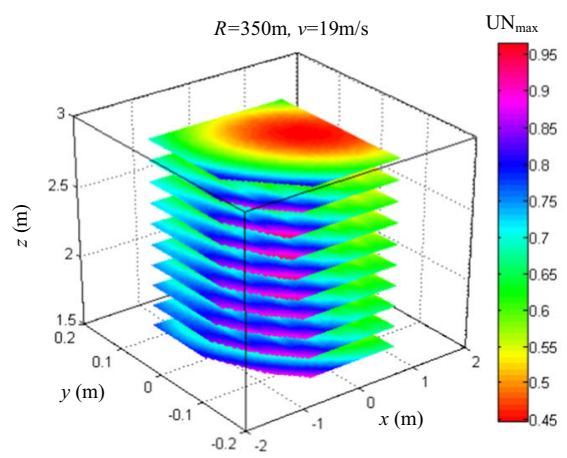

(c) NO.3 simulation case

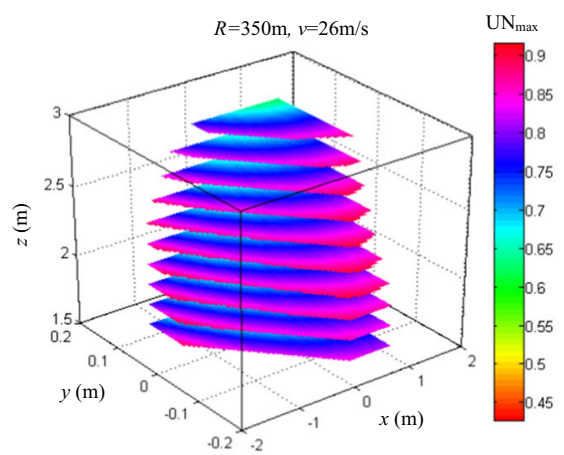

(e) NO.5 simulation case

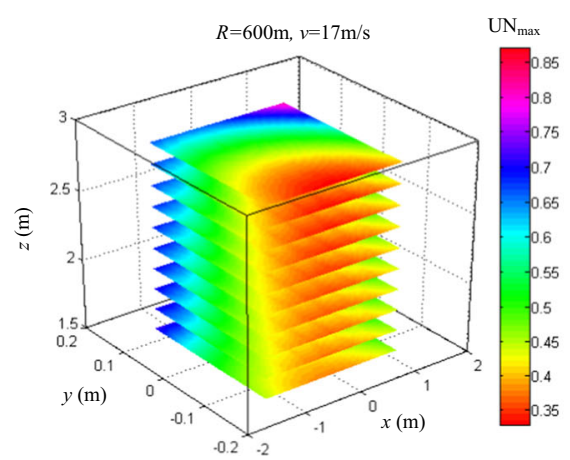

(g) NO.7 simulation case

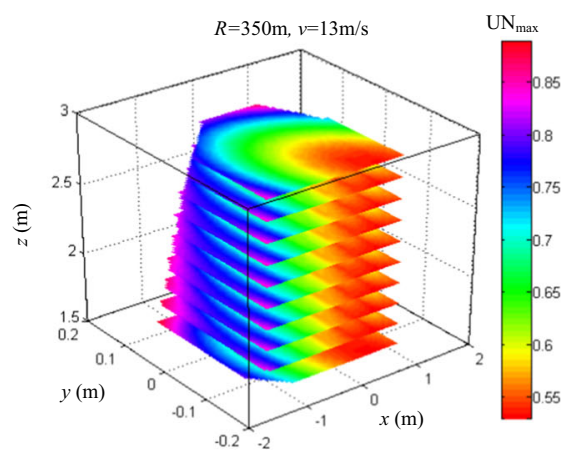

(b) NO.2 simulation case

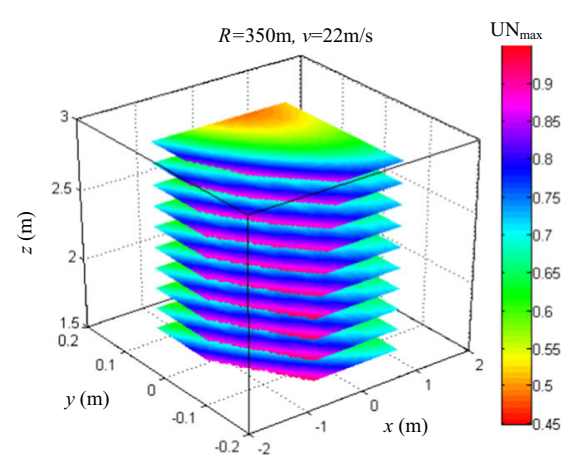

(d) NO.4 simulation case

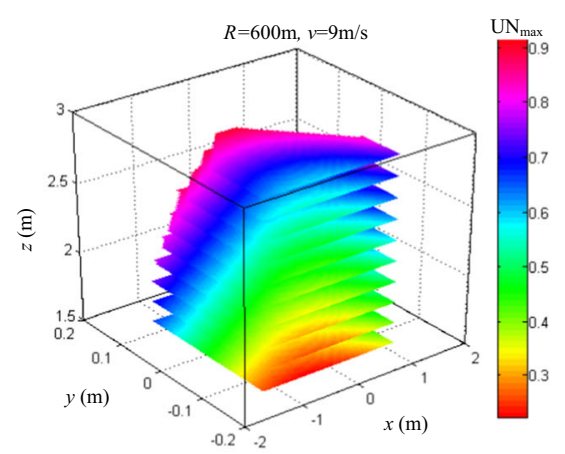

(f) NO.6 simulation case

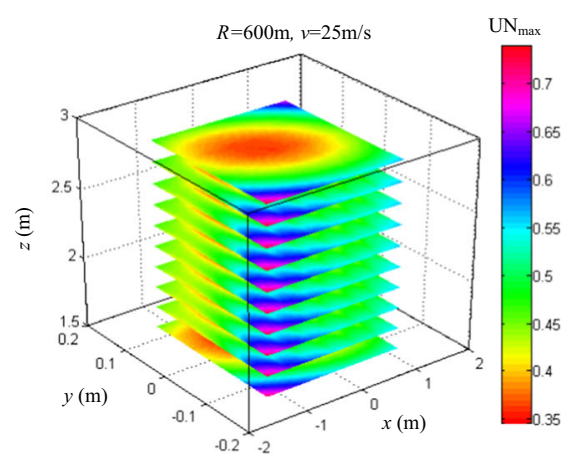

(h) NO.8 simulation case

Fig. 8 Distributions of $\mathrm{UN}_{\max }$ for the fitting data 


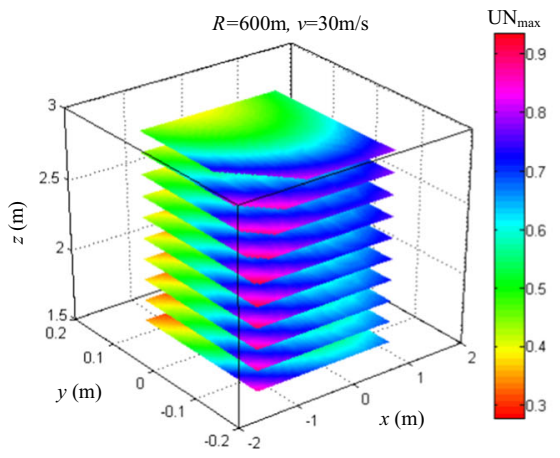

(i) NO.9 simulation case

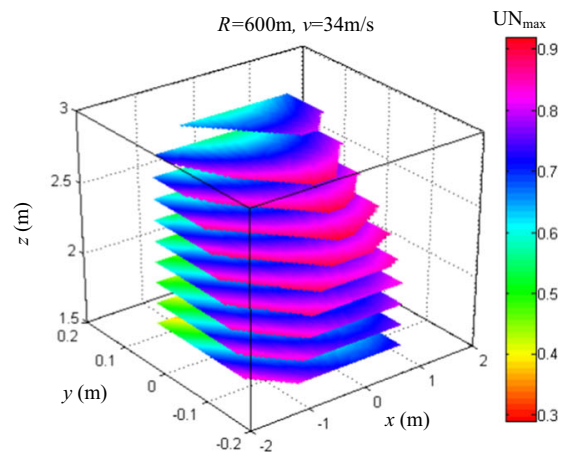

(j) NO.10 simulation case

Fig. 8 continued

Acknowledgements This work is supported by the National Natural Science Foundation of China [Grant Number U1834209]; the National Key Research and Development Program of China [Grant Number 2017YFB1200701]. The corresponding author is also supported by the China Scholarship Council.

Funding Open Access funding enabled and organized by Projekt DEAL.

\section{Declarations}

Conflict of interest The authors declare that they have no conflict of interest.

Open Access This article is licensed under a Creative Commons Attribution 4.0 International License, which permits use, sharing, adaptation, distribution and reproduction in any medium or format, as long as you give appropriate credit to the original author(s) and the source, provide a link to the Creative Commons licence, and indicate if changes were made. The images or other third party material in this article are included in the article's Creative Commons licence, unless indicated otherwise in a credit line to the material. If material is not included in the article's Creative Commons licence and your intended use is not permitted by statutory regulation or exceeds the permitted use, you will need to obtain permission directly from the copyright holder. To view a copy of this licence, visit http://creativecommons.org/licenses/by/4.0/.

\section{References}

1. Sun, Y.Q., Dhanasekar, M.: A dynamic model for the vertical interaction of the rail track and wagon system. Int. J. Solids Struct. 39, 1337-1359 (2002)

2. Zhai, W.M., Cai, C.B., Guo, S.Z.: Coupling model of vertical and lateral vehicle/track interactions. Veh. Syst. Dyn. 26, 61-79 (1996)
3. Diana, G., Cheli, F., Bruni, S., Collina, A.: Interaction between railroad superstructure and railway vehicles. Veh. Syst. Dyn. 23, 75-86 (1994)

4. Fröhling, R.D.: Low frequency dynamic vehicle/track interaction: modelling and simulation. Veh. Syst. Dyn. 29, 30-46 (1998)

5. Ripke, B., Knothe, K.: Simulation of high frequency vehicle-track interactions. Veh. Syst. Dyn. 24, 72-85 (1995)

6. Sinokrot, T., Nakhaeinejad, M., Shabana, A.A.: A velocity transformation method for the nonlinear dynamic simulation of railroad vehicle systems. Nonlinear Dyn. 51, 289-307 (2008)

7. Zhang, D., Tang, Y., Clarke, D.B., Peng, Q., Dong, C.: An innovative method for calculating diagonal lashing force of cargo on railway wagons in a curve alignment. Veh. Syst. Dyn. 59(3), 352-374 (2021)

8. Zboinski, K., Dusza, M.: Bifurcation analysis of 4-axle rail vehicle models in a curved track. Nonlinear Dyn. 89, 863-885 (2017)

9. Ye, Y., Zhang, Y., Wang, Q., Wang, Z., Teng, Z., Zhang, H.: Fault diagnosis of high-speed train suspension systems using multiscale permutation entropy and linear local tangent space alignment. Mech. Syst. Signal Process. 138, 106565 (2020)

10. Zboinski, K., Golofit-Stawinska, M.: Investigation into nonlinear phenomena for various railway vehicles in transition curves at velocities close to critical one. Nonlinear Dyn. 98, 1555-1601 (2019)

11. Gao, X., Li, Y., Yue, Y.: The "resultant bifurcation diagram" method and its application to bifurcation behaviors of a symmetric railway bogie system. Nonlinear Dyn. 70, 363-380 (2012)

12. UIC: Code of practice for the loading and securing of goods on railway wagons, Paris (2019)

13. Transportation Technology Center, Inc.: General rules covering loading of carload shipments of commodities in closed cars. Pueblo, CO (2010)

14. Railway, C.: General Rules Covering Loading and Reinforcement in Railway Freight Wagons. Chinese Railway Press, Beijing (2006)

15. Shatunov, O.V., Shvets, A.O.: Study of dynamic indicators of flat wagon with load centre shift. Science and transport 
progress. Bull. Dnipropetrovsk Natl. Univ. Railw. Transp. 2(80), 127-143 (2019)

16. Bao, T., Han, M., Chen, C., Chen, S.: Study on the relationship between lateral offset of the center of gravity of goods C70 gondola and the derailment coefficient. In: 2015 International Conference on Mechatronics, Electronic, Industrial and Control Engineering (MEIC-15). Atlantis Press (2015)

17. Matsumoto, A., Michitsuji, Y., Tobita, Y.: Analysis of trainoverturn derailments caused by excessive curving speed. Int. J. Railw. Technol 5, 27-45 (2016)

18. Bekele, D.: Effect of Railway Vehicle Parameters Variation on Derailment Running on Curved Track. AAU (2015)

19. Zhang, D., Clarke, D.B., Peng, Q., Gao, H., Dong, C.: Effect of the combined centre of gravity on the running safety of freight wagons. Veh. Syst. Dyn. 57, 1271-1286 (2019)

20. Suda, Y., Anderson, R.J., Yamada, K.: Dynamics of unsymmetric suspension trucks with semiactive control. Veh. Syst. Dyn. 23, 480-496 (1994)

21. Suda, Y., Grencik, J.: The mechanism of enhanced curving performance of unsymmetric suspension trucks under acting traction/brake torque. Veh. Syst. Dyn. 25, 629-640 (1996)

22. Keropyan, A.M., Kantovich, L.I., Voronin, B.V., Kuziev, D.A., Zotov, V.V.: Influence of uneven distribution of coupling mass on locomotive wheel pairs, its tractive power, straight and curved sections of industrial rail tracks. In: IOP Conference Series: Earth and Environmental Science. IOP Publishing (2017)
23. Ghazavi, M.R., Taki, M.: Dynamic simulations of the freight three-piece bogie motion in curve. Veh. Syst. Dyn. 46, 955-973 (2008)

24. Zhai, W.: Vehicle-Track Coupled Dynamics: Theory and Applications. Springer Nature, Berlin (2019)

25. CEN: BS EN 14363: 2016 Railway Applications-Testing and Simulation for the Acceptance of Running Characteristics of Railway Vehicles-Running Behaviour and Stationary Tests, London (2016)

26. CEN: BS EN 13803:2017 Railway Applications-TrackTrack Alignment Design Parameters-Track Gauges 1435 $\mathrm{mm}$ and Wider, London (2017)

27. Garg, V.: Dynamics of railway vehicle systems. Elsevier, Ontario (2012)

28. Peng, B., Iwnicki, S., Shackleton, P., Song, Y.: General conditions for railway wheel polygonal wear to evolve. Veh. Syst. Dyn. (2019). https://doi.org/10.1080/00423114.2019. 1697458

29. Zhang, D., Tang, Y., Sun, Z., Peng, Q.: Optimising the location of wagon gravity centre to improve the curving performance. Veh. Syst. Dyn. (2020). https://doi.org/10. 1080/00423114.2020.1865546

Publisher's Note Springer Nature remains neutral with regard to jurisdictional claims in published maps and institutional affiliations. 